Transcontinentales

continentales

Sociétés, idéologies, système mondial

$6 \mid 2008$

Mémoires et nations

\title{
Construction nationale et enjeux mémoriels
}

Jean-Luc Racine

\section{OpenEdition}

\section{Journals}

Édition électronique

URL : http://journals.openedition.org/transcontinentales/393

DOI : 10.4000/transcontinentales.393

ISBN : 978-2-7351-1561-7

ISSN : 1775-397X

Éditeur

Editions de la maison des sciences de l'homme

Édition imprimée

Date de publication : 30 juin 2008

ISBN : 978-2-200-92511-6

ISSN : 1950-1684

Référence électronique

Jean-Luc Racine, «Construction nationale et enjeux mémoriels », Transcontinentales [En ligne], 6 2008, mis en ligne le 06 avril 2011, consulté le 22 septembre 2020. URL : http://

journals.openedition.org/transcontinentales/393 ; DOI : https://doi.org/10.4000/transcontinentales. 393

Tous droits réservés 


\section{Construction nationale et enjeux mémoriels}

Comment, dans des sociétés multiculturelles, le processus de construction nationale prend-il en compte la pluralité des mémoires collectives, dont le statut est d'évidence très variable? L'arithmétique démographique pénalise souvent les petites communautés, mais parfois les dominants eux-mêmes sont minoritaires, et doivent leur position hégémonique à des rapports de force socioéconomiques, que confortent les mythes nationaux leur faisant la part belle. Dans la plupart des cas de figures, un constat s'impose: toutes les mémoires ne pèsent pas le même poids. Les études ici rassemblées témoignent toutefois d'une diversité qui ne se réduit pas à une simple dialectique opposant les dominés aux dominants, pour de multiples raisons. D'une part, tous les dominés ne sont pas soumis au même statut, et d'autre part, il arrive que les hiérarchies évoluent. Enfin, un processus quasi global anime aujourd'hui, dans maints pays, les minorités qui revendiquent leur part du passé national et une reconnaissance nouvelle. La volonté politique qui préside au processus de construction nationale peut dès lors évoluer, quand il apparaît prudent, ou nécessaire, de redéfinir la nation en donnant une place accrue à sa diversité, et aux passés qui l'ont forgée. Derrière les luttes mémorielles, les enjeux de pouvoir ne sont pas seuls en cause. Comptent aussi la quête de dignité, la volonté de voir reconnaître les préjudices subis au fil de l'histoire, ou, plus simplement, le droit à être soi, et néanmoins égal aux autres.

De la vingtaine d'interventions présentées au premier colloque des instituts français de recherche à l'étranger, qui s'est déroulé au musée du quai Branly fin $2007^{1}$, quatre ont été retenues ici, qui, chacune à leur façon, posent le problème de la relation entre construction nationale et enjeux mémoriels. Le dossier proposé dans ce numéro de Transcontinentales illustre ainsi en Amérique latine, en Asie, dans le monde arabe et dans les Mascareignes, aux portes de l'Afrique, des rapports à l'histoire fort divers, encore que certains thèmes transversaux se dégagent parfois, comme celui des effets des migrations, forcées ou non, sur les processus de construction nationale ou la place, variable, de l'ethnicité dans les sociétés multiculturelles.

1 - Le programme du colloque «Présences du passé» et les résumés de toutes les interventions sont disponibles sur le site www.ifre.fr, ainsi que l'enregistrement vidéo de l'ensemble des sessions, session inaugurale et table ronde de clôture incluses. La conférence inaugurale de Marie-Claire Lavabre a été publiée dans le numéro précédent de Transcontinentales sous le titre "Paradigmes de la mémoire» (Transcontinentales, $\mathrm{n}^{\circ} 5,2^{\mathrm{e}}$ semestre 2007, p. 139-147). 
Autre fil conducteur: comment les constructions nationales prennent-elles en compte, face au discours de l'État, la pluralité des mémoires collectives? La question est explicitement posée dans plusieurs des contributions. Elle renvoie aux rapports entre le multiculturalisme affiché - le modèle mauricien d' "unité dans la diversité», ou les discours officiels mexicains ou colombiens sur la diversité culturelle - et la réalité des relations sociales et de la distribution du pouvoir. Cette discordance entre discours étatique(s) dominant(s) et perceptions "vues d'en bas » se retrouve, dans un autre contexte, dans l'étude de la mémoire (des mémoires) de la partition de l'Inde et du Pakistan. Enfin, un autre paramètre de la question mémorielle, celui de l'oubli et du silence, est abordé. Oblitération délibérée de la part des nouveaux maîtres de la République yéménite cherchant à gommer de l'histoire nationale le règne de leurs prédécesseurs, quitte à englober dans un même silence des dynasties lointaines. Oubli officiel, en Inde et au Pakistan, des massacres de la Partition, les gouvernements ne célébrant pas la mémoire des victimes pour mieux mettre en avant l'avancée majeure que fut l'indépendance nationale.

Les ruses de la mémoire sont donc infinies. Elles cherchent, à tous les niveaux de la nation, fût-ce de façons contrastées, à faire des choix pour mettre en avant des vérités partielles ou réinventées, afin de définir ou de redéfinir les appartenances identitaires, dans une tension constante entre la voix officielle de la nation, portée par l'État - dans son incarnation du moment -, et les fragments de la nation que sont les communautés faisant entendre leur voix pour mieux trouver place dans la mémoire nationale, qui n'est jamais figée.

\section{Registres mémoriels et ethnicité : I'île Maurice}

L'île Maurice, le plus petit des États étudiés ici, n'est pas pour autant le moins complexe, puisqu'une population très diverse s'y est fixée, en plusieurs étapes. D'abord des Franco-Mauriciens, "Mauriciens blancs d'origine française», le premier groupe à construire une mémoire communautaire nourrie de l'histoire coloniale de la mise en valeur de l'île. Puis les descendants d'esclaves, baptisés Créoles, groupe marginalisé qui tente aujourd'hui de «réinventer son africanité». Enfin les migrants venus de l'Inde, et pour la plupart hindous : pour beaucoup des coolies engagés après la suppression de l'esclavage. Cette population d'origine indienne est aujourd'hui à la tête du pouvoir politique. Dans un tel système «qui pose la communauté ethnique et sa mémoire comme fondement principal de la légitimité de l'accès aux ressources de l'État», Catherine Boudet et Julie Peghini s'interrogent sur les nouvelles dynamiques identitaires qui se font jour et sur les modalités "d'émergence d'une mémoire collective» qui donnerait à tous une même nation, en récusant la "permanence d'un triptyque mémoriel» fondé sur les grandes catégories historico-ethniques. En effet, "l'antériorité comme mode de légitimation» ne peut plus suffire : l'hégémonie des «hindous» en témoigne, tandis que le "processus de réhabilitation des mémoires des dominés» n'est pas seulement mené pour reconstruire une image valorisante de ceux qui furent marqués par l'esclavage, et qui surent aussi chercher la liberté. Il s'inscrit également dans des "rapports de concurrence entre registres mémoriels», qu'illustrent parfaitement la compétition entre «lieux de mémoire» et les messages portés par divers musées. La concurrence entre le groupe indien, pour qui le 
«moment-origine» est celui de l'indépendance de l'île, et le groupe franco-mauricien, pour qui la colonisation fut le «moment-origine», s'enrichit aujourd'hui de «la réappropriation de la mémoire historique des "sans-voix" ». Et peut-être cela pourra-t-il étayer le "travail de fondation d'une identité nationale» qu'aiderait "l'émergence d'une mémoire nationale communément partagée», pas encore acquise.

\section{Au-delà de l'ethnicité : \\ "entre-soi " et "entre deux" au Mexique et en Colombie}

Dans deux pays d'Amérique latine, Anath Ariel de Vidas et Odile Hoffmann mettent en lumière une autre logique, qui ne fait pas la part aussi belle à l'ethnicité. Indiens teenek et nahua du Mexique et populations noires du Mexique et de Colombie sont tous des communautés dominées, mais l'histoire des origines varie. Les Indiens peuvent se prévaloir de l'antériorité qui découle, par définition, de l'autochtonie, qui a trouvé place dans la légitimation du discours national - mais un des deux groupes a migré au $\mathrm{xx}^{\mathrm{e}}$ siècle -, alors que les populations noires sont associées à l'esclavage, dévalorisant, même si certains groupes revendiquent une liberté originelle, résultat du naufrage d'un bateau négrier, permettant d'arriver libre sur les terres nouvelles. Au-delà des temporalités diverses et des affichages ethniques, les auteurs mettent en avant d'autres concepts, d'autres clés d'analyse. La comparaison des ressources narratives et des bagages mémoriels des différents groupes conduit à nuancer le concept d'autochtonie, car «[d]ans aucun des cas mentionnés, le critère $\mathrm{d}^{\prime \prime}$ autochtonie" " n'est activé localement dans l'acception donnée par les catégories de l'État (Indiens versus "Noirs"). En revanche, il semble fonctionner si on lui accorde le sens élaboré par les populations locales pour fonder l'identité collective sur un rapport subjectif à l'origine». Se dévoilent ainsi deux catégories qui transcendent les appartenances ethniques : celle de "l'entre-soi » caractérise les "collectifs régionaux installés de longue date», comme les Indiens teenek ou les "Noirs» du Pacifique colombien, alors que "l'entre-deux» marque "des groupes à l'ancrage territorial récent ou contesté par un environnement socio-ethnique distinct», tels les Indiens nahua ou les villages noirs de la Costa Chica. Pour les premiers, la mémoire s'inscrit dans les mythes ancrés dans une grande profondeur historique : «Le recours à l'histoire s'entremêle au temps mythique pour expliquer le présent. » Chez les seconds, "[l]e dispositif mémoriel s'élabore a posteriori, à partir des matériaux glanés dans les mémoires individuelles et ceux qui sont mis à disposition par le contexte historique du moment, le gouvernement, les institutions et le récit national ». Il faudra bien que ce récit national - ou ces récits nationaux - fasse place à cette diversité des «modalités de la mémoire collective», très contextualisée par les groupes eux-mêmes. Cette démarche ne peut se réduire à la "gestion politique de la multiethnicité qui fait appel à l'autochtonie différentielle des groupes».

\section{Effacer mille ans au Yémen?}

En 1962, au Yémen, la proclamation de la République porte au pouvoir une nouvelle classe sociale, dont les dirigeants, chefs tribaux et juges, récusent la dernière dynastie des imams zaydites - tenants d'une variante du chiisme. Cas de figure plutôt classique, si ce n'est que l'occultation du passé n'englobe pas seulement la dernière 
dynastie au pouvoir, mais près de mille ans d'histoire au fil desquels diverses dynasties d'imams zaydites ont régné. Il faut dès lors revaloriser le passé pré-islamique - ce que rend possible l'évocation de la reine de Sabâ -, ou redécouvrir un historien et géographe du $\mathrm{x}^{\mathrm{e}}$ siècle, al-Hamdânî, quitte à lui attribuer des ouvrages bien plus tardifs. Jean Lambert dénoue ici les fils d'une histoire complexe : histoire politique, histoire intellectuelle, histoire sociale, sur laquelle se greffent les paramètres géographiques, les appartenances tribales, les compétitions entre Arabes du Nord et Arabes du Sud. Dans ce travail de réécriture (ou, ici encore, d'occultation) de l'histoire, c'est bien une conception de la nation qui en affronte une autre. Mais, demande l'auteur, "comment construire l'identité contemporaine du Yémen sans la composante culturelle du zaydisme et son patrimoine intellectuel si original et si marquant»? En jouant la carte d'un panislamisme d'inspiration sunnite, ou celle d'une "arabisation sur un mode nationaliste»? En redécouvrant al-Hamdânî ou en célébrant l'antiquité du pays, les nouvelles élites cherchent à redéfinir de nouvelles filiations, en ouvrant «cette porte vers un modèle légendaire à un islam venu du nord». Et Jean Lambert de conclure, devant ce chantier idéologique inachevé, que les compétitions mémorielles du Yémen contemporain illustrent un processus par lequel «à l'écriture d'une histoire dominante, succède non pas l'écriture d'une histoire des opprimés, mais celle d'une nouvelle histoire dominante».

\section{Inde et Pakistan : partition de la mémoire}

La partition de l'empire des Indes, qui donne naissance à l'Inde et au Pakistan en 1947, résulte de l'antagonisme entre deux conceptions opposées de la nation : multireligieuse et multiculturelle pour l'Inde; fondée sur l'identité religieuse pour le Pakistan, qui s'affirme comme la terre des musulmans indiens. Cette partition ne fut pas simplement négociée entre la puissance britannique sur le départ et les deux principaux partis, le Congrès et la Ligue musulmane. Elle fut aussi conduite dans le sang des massacres, et elle a suscité un mouvement de réfugiés de très grande ampleur. Lionel Baixas s'attache ici à éclairer comment, après l'indépendance de l'Inde et du Pakistan, des stratégies d'oubli ou d'occultation ont longtemps prévalu. Oubli - ou silence - de ceux qui furent pris dans ce tourbillon de l'histoire. Discrétion des autorités, qui célèbrent l'indépendance sans en rappeler le traumatisme, l'histoire "orthodoxe» de la Partition contribuant à «éluder sa réalité» humaine. La chercheuse pakistanaise Ayesha Jalal peut ainsi constater que «la réalité psychologique de la partition a laissé de bien plus profondes cicatrices dans l'esprit des populations que les dynamiques sociales, économiques et politiques qui ont conduit à la division ». Les choses bougent cependant : par le biais d'une nouvelle génération d'historiens ou d'intellectuels qui collectent les témoignages des derniers survivants, ou qui appellent à réviser les manuels scolaires. Par l'engagement d'artistes et de gens de théâtre indiens et pakistanais, qui s'approprient désormais ce moment de division pour repenser ensemble ce que fut la Partition. Désormais sollicitées, mémoires individuelles et mémoires collectives aident à renouveler la lecture de l'histoire et à dépasser la «tyrannie du silence» qui mettait en avant ce qui sépare, plutôt que ce qui fut commun, fût-ce dans l'épreuve et la douleur. La mémoire retrouvée apparaît ainsi "comme catharsis», et comme moyen de "faire la paix avec la Partition». Ce qui est sans doute le préalable indispensable à la paix tout court, sinon à la réconciliation. 


\section{La France et la mémoire postcoloniale}

À partir de son intervention dans le débat de clôture du colloque «Présences du passé», Marie-Claude Smouts livre ici une réflexion sur «la frilosité française» vis-à-vis de la question postcoloniale qui, abordée avec retard par rapport aux pays anglo-saxons, est fréquemment soumise à une double interrogation critique. Comment construire épistémologiquement cet objet, dès lors qu'il quitte le champ académique pour prendre corps dans le débat public, et comment faire en sorte que la revendication mémorielle ne s'enferme pas "dans une logique binaire ("nous" et "eux")» lourde de conséquences possibles sur le tissu national et sur le jeu politique? Jugeant pour sa part que les revendications mémorielles traduisent en fait un désir d'intégration, Marie-Claude Smouts constate combien le champ social s'est trouvé, en l'affaire, en avance sur le champ politique, voire sur la recherche. Mais elle prend bien garde de souligner qu'il importe que la construction de la mémoire coloniale ne vire pas à «la guerre des mémoires», qu'elle n'exige pas «une quelconque repentance», et qu'elle ne soit pas instrumentalisée.

\section{Marc Ferro, le passé, le présent}

Dans l'entretien qui couronne ce dossier, Marc Ferro revient sur ce qui fut une ligne de force de nombre de ses travaux d'historien : les liens entre passé et présent et, en particulier, la force du ressentiment, qu'il identifie comme étant l'un des moteurs de l'histoire. Son expérience de l'Algérie, où il enseigna dans les années 1950, et son premier champ de recherche, l'URSS, dessinent le parcours d'un intellectuel qui fut tenté par la politique avant de se livrer tout entier à la passion de l'histoire. L'expérience algérienne et l'histoire soviétique permettent aussi d'aborder avec recul de grandes questions sur les rapports entre le pouvoir et l'écriture de l'histoire, de la définition la "vérité historique» - fluctuante au besoin - par le parti unique des États totalitaires jusqu'aux lois mémorielles qui agitent la France d'aujourd'hui. Au passage, le débat s'ouvre sur les modes de résolution des conflits, et sur les différents périmètres des négociations qui regroupent, comme ce fut le cas in fine en Irlande, modérés et extrémistes, ou qui s'illusionnent en recherchant exclusivement des «interlocuteurs valables», au risque d'ignorer les opposants modérés pour finir par traiter avec les radicaux, comme ce fut le cas en Algérie. Israël, Palestine, Afrique du Sud retiennent tour à tour l'attention. Au cœur de la dialectique passé/présent s'inscrivent aussi les travaux de Marc Ferro sur «comment on raconte l'histoire aux enfants» et le rôle novateur qui fut le sien en démontrant, des années durant, la capacité du cinéma à éclairer l'histoire, ou la «contre-histoire».

\section{Varia}

\section{La Banque asiatique de développement}

On sait combien la querelle mémorielle peut tendre les relations sino-japonaises, quand le souvenir des massacres de Nankin ou la célébration des «morts pour la patrie» au sanctuaire de Yasukuni illustrent, en parfaite antithèse, des visions opposées de l'histoire. L'article de Guy Faure, consacré à la Banque asiatique de 
développement (BAD), offre une image plus nuancée des relations entre Japon et Chine, et de la subtilité des jeux d'influences dans une institution où les ÉtatsUnis n'endentent pas se voir marginalisés. La Banque, où le Japon bénéficie depuis l'origine d'une influence considérable et monopolise les présidences successives de l'institution, affiche un triple objectif, au bénéfice des pays d'Asie : croissance économique durable, développement social, bonne gouvernance. Cet affichage n'exclut naturellement pas les critiques au vu des résultats, mais du moins la Banque peut-elle se prévaloir de quelques atouts. D'une part, elle "constitue une exception, celle de ne pas être sous la "tutelle occidentale" mais sous celle, incontestée, du Japon", qui cherche à promouvoir le principe du consensus. En second lieu, elle a fait preuve d'une capacité à conduire des programmes de coopération économique bien plus efficacement que l'ASEAN n'a su le faire. Le meilleur exemple en est la région du Grand Mékong, où un programme phare de la coopération régionale de la BAD associe pour l'aménagement du bassin du fleuve cinq États riverains (Cambodge, Laos, Vietnam, Myanmar, Thaïlande) et deux provinces chinoises (Yunnan et Guangxi). Cette opération illustre comment la Chine, qui a rejoint la Banque en 1986, a su jouer de ce que Guy Faure appelle "un multilatéralisme intéressé», pour prendre du poids dans la Banque, en déployant une stratégie de coopération qui cherche à dissiper la perception d'une menace chinoise «à tous niveaux, qu'ils soient stratégiques, économiques ou politiques». Un nouveau style de diplomatie est dès lors mis en œuvre, qui illustre bien comment la Chine travaille, en particulier en Asie, mais aussi ailleurs, à réussir «son insertion dans le système international et dans ses organismes multilatéraux» et ce, tout en menant son "grand jeu géopolitique», comme elle le fait autour du Grand Mékong. En définitive, une certaine "convergence diplomatique» entre le Japon et la Chine n'est pas impossible à moyen terme. L'hypothèse japonaise d'un fonds monétaire asiatique spécifique, rejetée par Washington, pourrait offrir un champ de convergence entre Tokyo et Pékin, qui reste pour l'heure, sur cette question, dans l'expectative. Si la Chine y voyait finalement intérêt, pour aider à contrer les crises financières ou les tensions sur les monnaies, elle aurait assurément les moyens d'apporter un capital considérable à une initiative qui conforterait, là encore, la montée en puissance de l'Asie.

\section{La microfinance : un bilan mitigé}

C'est un autre volet des circuits financiers qu'analysent, pour clore ce numéro, Isabelle Guérin, Cyril Fouillet et Marc Roesch, en dressant le bilan des opérations de microfinance en Inde, un pays où ce mode de financement en direction de populations généralement hors des circuits bancaires établis connaît un essor considérable, principalement à destination des femmes. Cet essor, toutefois, n'est pas seulement le résultat d'initiatives venues d'ONG au service des plus défavorisés. En s'attachant à l'exemple du Tamil Nadu - un État de l'Inde où la microfinance a beaucoup progressé - les auteurs mettent en lumière l'intérêt des banques pour ce système. Avec l'encouragement de l'État, une relation s'est tissée entre banques, ONG et groupes impliqués (des "groupes se prenant en mains" : self-help-groups). Pour des effets jugés mitigés : "si les résultats peuvent être considérés comme positifs d'un point de vue quantitatif (nombre de clients, volume de crédits et taux de remboursement), la qualité des services reste très médiocre», et la microfinance "se révèle 
incapable de modifier les contraintes structurelles». Certes on peut constater des avancées (meilleure gestion de la trésorerie familiale, financement des dépenses de santé et d'éducation), mais cette voie laisse entière, en termes d'emploi, «la question de la diversification des économies rurales», et n'a pas de résultat significatif en termes de diminution de la pauvreté ou des inégalités. Face à la finance formelle - le système bancaire - la microfinance ne peut pallier les effets de la libéralisation. Face à la finance informelle - les prêteurs ou les usuriers - elle ne peut offrir la même souplesse, ni une totale alternative. En définitive, le développement des liens entre banques, ONG et groupes emprunteurs a permis «un développement massif de l'offre de microfinance»; mais "concilier offre de masse et qualité des services s'avère beaucoup plus problématique», comme en témoigne à maints égards un fin travail d'enquêtes de terrain.

Jean-Luc Racine 